\title{
International Myeloma Working Group risk stratification model for smoldering multiple myeloma (SMM)
}

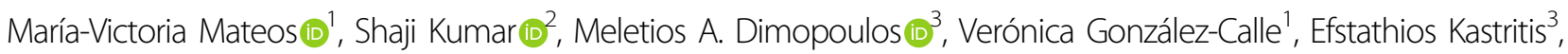

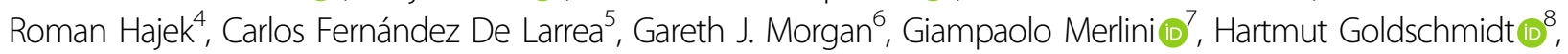 \\ Catarina Geraldes 9 , Alessandro Gozzetti (10 ${ }^{10}$, Charalampia Kyriakou ${ }^{11}$, Laurent Garderet ${ }^{12}$, Markus Hansson (1) ${ }^{13}$, \\ Elena Zamagni ${ }^{14}$, Dorotea Fantl ${ }^{15}$, Xavier Leleu ${ }^{16}$, Byung-Su Kimm ${ }^{17}$, Graça Esteves ${ }^{18}$, Heinz Ludwig ${ }^{19}$, Saad Usmani $\mathbb{1}^{20}$, \\ Chang-Ki Min ${ }^{21}$, Ming Qi2 ${ }^{22}$, Jon Ukropec ${ }^{22}$, Brendan M. Weisss ${ }^{22}$ S. Vincent Rajkumar $\mathbb{E}^{2}$, Brian G. M. Durie ${ }^{23}$ and \\ Jesús San-Miguel $\mathbb{B}^{24}$
}

\begin{abstract}
Smoldering multiple myeloma (SMM) is an asymptomatic precursor state of multiple myeloma (MM). Recently, MM was redefined to include biomarkers predicting a high risk of progression from SMM, thus necessitating a redefinition of SMM and its risk stratification. We assembled a large cohort of SMM patients meeting the revised IMWG criteria to develop a new risk stratification system. We included 1996 patients, and using stepwise selection and multivariable analysis, we identified three independent factors predicting progression risk at 2 years: serum M-protein $>2 \mathrm{~g} / \mathrm{dL}$ ( $H R$ : 2.1), involved to uninvolved free light-chain ratio $>20$ (HR: 2.7), and marrow plasma cell infiltration $>20 \%$ (HR: 2.4). This translates into 3 categories with increasing 2-year progression risk: $6 \%$ for low risk (38\%; no risk factors, HR: 1); 18\% for intermediate risk (33\%; 1 factor; HR: 3.0), and 44\% for high risk (29\%; 2-3 factors). Addition of cytogenetic abnormalities $(\mathrm{t}(4 ; 14), \mathrm{t}(14 ; 16),+1 \mathrm{q}$, and/or del13q) allowed separation into 4 groups (low risk with 0 , low intermediate risk with 1 , intermediate risk with 2, and high risk with $\geq 3$ risk factors) with 6, 23, 46, and 63\% risk of progression in 2 years, respectively. The $2 / 20 / 20$ risk stratification model can be easily implemented to identify high-risk SMM for clinical research and routine practice and will be widely applicable.
\end{abstract}

\section{Introduction}

Smoldering multiple myeloma (SMM) represents a transitional stage between monoclonal gammopathy of undetermined significance (MGUS) and active multiple myeloma $(\mathrm{MM})^{1,2}$. Before International Myeloma Working Group (IMWG) revised the MM diagnostic criteria,

\footnotetext{
Correspondence: Shaji Kumar (Kumar.Shaji@mayo.edu)

${ }^{1}$ Servicio de Hematologia, Hospital Universitario de Salamanca, Centro de Investigación del Cáncer, Instituto de Biología Molecular y Cellular del Cáncer (Universitario de Salamanca-Consejo Superior de Investigaciones Científicas), Salamanca, Spain

2Division of Hematology, Mayo Clinic, Rochester, MN, USA

Full list of author information is available at the end of the article

These authors contributed equally: María-Victoria Mateos, Shaji Kumar
}

SMM was defined by the presence of either a serum monoclonal protein of $\geq 3 \mathrm{~g} / \mathrm{dL}$ (or $\geq 500 \mathrm{mg} / 24 \mathrm{~h}$ in urine or both) and/or $\geq 10 \%$ bone marrow plasma cells (BMPCs) without evidence of any CRAB symptoms (hypercalcemia, renal impairment, anemia, or lytic bone lesions). SMM is a heterogeneous disease with a risk of progression to MM of $10 \%$ per year during the first 5 years following diagnosis, decreasing to $3 \%$ per year over the subsequent 5 years, and $1 \%$ per year after the 10 years of diagnosis ${ }^{3}$. The Mayo Clinic and the Spanish Group had previously created different risk stratification models that can identify SMM patients with a 2-year risk of progression to MM of $\geq 50 \%$. The Mayo Clinic model included M-protein $(\geq 3 \mathrm{~g} / \mathrm{dL})$,

\section{(c) The Author(s) 2020}

(c) (i) Open Access This article is licensed under a Creative Commons Attribution 4.0 International License, which permits use, sharing, adaptation, distribution and reproduction in any medium or format, as long as you give appropriate credit to the original author(s) and the source, provide a link to the Creative Commons license, and indicate if changes were made. The images or other third party material in this article are included in the article's Creative Commons license, unless indicated otherwise in a credit line to the material. If material is not included in the article's Creative Commons license and your intended use is not permitted by statutory regulation or exceeds the permitted use, you will need to obtain permission directly from the copyright holder. To view a copy of this license, visit http://creativecommons.org/licenses/by/4.0/. 
BMPCs infiltration $(\geq 10 \%)$, and the ratio of serum free light chain $(\mathrm{sFLC})(\geq 8$ or $<0.125)$ to categorize patients into three subgroups, with a $52 \%$ risk of progression in 2 years for those presenting with all three risk factors ${ }^{4}$. The Spanish model used the presence of $\geq 95 \%$ clonal plasma cells among all BMPCs by immunophenotyping and the presence of immune paresis and identifies a subgroup of patients having both features with a $50 \%$ risk of progression at 2 years ${ }^{5}$.

The standard of care for SMM, irrespective of the risk status of the patients, has been observation. However, there are two randomized studies confirming the benefit of early treatment in reducing the risk of progression to MM. The Spanish Myeloma Group conducted the first phase III trial in high-risk SMM, comparing lenalidomide plus dexamethasone $(\mathrm{Ld})$ versus observation, and Ld significantly delayed the progression to $\mathrm{MM}$ and patients lived longer (overall survival $(\mathrm{OS}))^{6}$. The Eastern Cooperative Oncology Group (ECOG) group has also reported a significant benefit for the early treatment with singleagent lenalidomide versus observation in $\mathrm{SMM}^{7}$.

Although the Spanish trial did not lead to a change in the standard of care, it encouraged the IMWG to revise the criteria for the definition of SMM and MM to identify a subset of SMM with a 2-year risk of progression of approximately $80 \%$ who were then categorized as MM. The identification of this subgroup is based on the presence of any one of these biomarkers: BMPCs $\geq 60 \%$ or involved/uninvolved sFLC ratio $\geq 100$, and presence of $>1$ focal lesion on magnetic resonance imaging, in patients having bone marrow with at least $10 \%$ of clonal BMPC infiltration. This led to a paradigm shift as these patients were then regarded as having myeloma and were offered therapy $^{8}$.

Other groups, in parallel, also created different models to identify SMM patients with a progression risk at 2 years of $50 \%$ using different features like positive uptake on positron emission tomography-computed tomography (PET-CT), type of M-protein, cytogenetic abnormalities, increase in serum M-protein and decrease in hemoglobin count over time, presence of Bence Jones proteinuria, or genetic signatures, among others ${ }^{9-14}$. At the same time, many clinical trials are ongoing in high-risk SMM according to different risk models, and all these developments brought to the fore two issues: (i) the heterogeneity of the different risk models developed using rather small series of SMM patients and (ii) the real impact of the revised SMM definition. This forms the basis of the current study.

We studied a large cohort of SMM patients to identify factors that predicted progression to MM with the goal of developing an easy risk score to predict the 2-year progression risk. The factors identified were M-protein ( $>2 \mathrm{~g} /$ $d L)$, BMPCs infiltration (>20\%), and the ratio of involved versus uninvolved sFLC $(>20)$. In addition, we have also created a risk score that accounts for the entire range of the variable measurements, which can be used to provide a more individualized 2-year risk of progression for every SMM patient.

\section{Patients and methods}

Patients with SMM diagnosed after January 2004 were included in this retrospective medical chart review from participating IMWG sites globally. The SMM definition required to be included in the study was based on the 2014 IMWG criteria ${ }^{8}$. Patients included in this analysis required baseline data from diagnosis $(+/-3$ months), no progression to $\mathrm{MM}$ or other plasma cell disorders within 6 months from diagnosis, a minimum follow-up of 1 year, and should not have been included in any therapeutic trial.

Trained staff collected data from the patient medical charts in three case report forms: (i) patient registration and demographics form, (ii) diagnosis and baseline assessment form, and (iii) 12-month assessment form. The Institutional Review Board at each site approved the study. The study was conducted in accordance with the Declaration of Helsinki and the International Council for Harmonisation Guidelines.

The primary objective was to develop a risk stratification model that will identify SMM patients who have high risk of progression to $\mathrm{MM}$ or other plasma cell disorders (50\% progression risk within the first 2 years from diagnosis) based on the 2014 IMWG criteria for definition of both SMM and MM.

Time to progression (TTP) to MM or amyloidosis was the primary end point and was defined as the time elapsed between diagnosis of SMM and when the patient experienced progressive disease. All patients who did not progress at the time of last follow-up were censored in the TTP analysis. A secondary objective was to create a risk scoring tool that would allow individualized estimate of risk at various time points from diagnosis.

\section{Statistical methods}

Due to the nature of the study, no formal hypothesis was used to calculate the sample size. We summarized categorical variables as proportions and continuous variables as medians (range). Univariate Cox regressions were run for each factor to assess an initial relationship between a factor and progression to $\mathrm{MM}$ or related disorders. For each factor where $p \leq 0.25$, optimal cut points were identified using Youden's Index. Receiver operating characteristic (ROC) curves were generated, with the cutoff maximizing both sensitivity and specificity determined. Sensitivity and specificity were reported for these optimal cut points. Interaction term between candidate variables were included in the model selection if candidate 
variables with correlations satisfy the above criteria. Using the optimal cut points, a stepwise regression analysis was used to determine predictive variables using a required selection stay criterion of 0.001 . A random forest algorithm consisting of an ensemble of decisions trees ${ }^{15}$ created using the random Forest package was used to confirm variable selection ${ }^{16}$. Important prognostic variables for predicting probability of progression at 2 years were identified and ranked based on the percentage change in mean squared error. Similar methods were used to identify the most significant cytogenetic abnormalities associated with progression to MM in patients with available information.

Patients were categorized based on the number of risk categories present and Cox proportional hazard regression models were used to calculate hazard ratios (HRs) and $95 \%$ confidence intervals (CIs). Kaplan-Meier curves were used to illustrate TTP across the number of risk categories present.

A risk score was developed to elucidate the potential strength of individual relationships between each of the previously defined variables based on the method described by Sullivan et al. ${ }^{17}$. Variables were categorized based on clinically relevant as well as spline functions to establish the general trends in the risk of progression with increasing laboratory values. Scores for each risk factor were assigned relative weights of each coefficient in the multivariable regression model. Predicted risk of progression were summarized based on this scoring tool. Analyses were conducted in SAS version 9.4 (The SAS Institute, Cary, NC) and R Core Team (2018).

\section{Results}

\section{Patient characteristics}

One thousand nine hundred and ninety-six SMM patients from 75 centers in 23 countries were included. Baseline characteristics are in Table 1 . The median age at diagnosis was 64 years (interquartile range (IQR): 56-72). The median serum M-component and BMPC infiltration were $1.8 \mathrm{~g} / \mathrm{dL}$ (IQR: 1.1-2.6) and 15\% (IQR: 12-25), respectively. Concerning cytogenetic information (available in 689 patients), trisomies and del13q/monosomy 13 were the most frequent abnormalities $(31.4 \%$ and $28.4 \%$, respectively). 1q gain was reported in $25.4 \%$ and $t(4 ; 14)$ and $\operatorname{del}(17 \mathrm{p} /$ monosomy 17$)$ in $10.2 \%$ and $5.4 \%$, respectively.

\section{Survival outcomes}

The median follow-up from diagnosis was 3.0 years (IQR 1.6-5.1). At the data cut-off, 815 (41\%) patients had progressed to MM or a related disorder. The median TTP for the entire cohort was 6.4 years (95\% CI 6.0-7.2); the 2-, 5 -, and 10-year risk of progression were 22,42 , and $64 \%$, respectively (Fig. 1). The estimated 5 - and 10-year OS for
Table 1 Baseline characteristics of the study population.

\begin{tabular}{lcc}
\hline & $\begin{array}{l}\text { Missing data } \\
(\boldsymbol{n})\end{array}$ & $\begin{array}{l}\text { Quantity, median } \\
\text { (IQR) or \% }\end{array}$ \\
\hline Age (years), median (range) & 0 & $64(56-72)$ \\
Gender (Male), \% & 0 & $978(49 \%)$ \\
Hemoglobin (g/dL) & 54 & $12.9(11.8-13.8)$ \\
Creatinine (mg/dL) & 136 & $0.88(0.71-1.04)$ \\
Calcium (mg/dL) & 172 & $9.3(9-9.7)$ \\
Albumin (g/dL) & 179 & $4(3.7-4.3)$ \\
Beta-2 microglobulin (mg/dL) & 357 & $2.3(1.8-3)$ \\
Serum M-protein (g/dL) & 0 & $1.8(1.1-2.6)$ \\
Serum FLC ratio & 633 & $6.2(2.1-24.1)$ \\
Heavy-chain type & 120 & \\
IgA & & $452(24.1 \%)$ \\
IgD & & $6(0.3 \%)$ \\
IgG & & $1402(74.7 \%)$ \\
IgM & & $16(0.9 \%)$ \\
Light-chain type & & \\
Kappa & 24 & $1199(60.8 \%)$ \\
Lambda & & $773(39.2 \%)$ \\
Immunoparesis ${ }^{a}$ & & $992-25)$ \\
Urine M-spike (mg/24 h) & $792.3 \%)$ \\
aspirate \% higher of biopsy and & 0 & \\
\hline & & \\
\hline
\end{tabular}

Immunoparesis was defined as reduction in one or more of the uninvolved immunoglobulin level below normal range.

${ }^{\mathrm{b}}$ Bone marrow plasma cell percentage.

the entire cohort from SMM diagnosis was 93.8\% (92.0-95.2) and 88.3\% (84.8-91.1), respectively.

\section{Risk factors for progression and stratification model}

The factors initially included in the univariate analysis for predicting progression risk at 2 years from the diagnosis are shown in Table 2 indicating those included in the model because of their capacity for predicting risk of progression to $\mathrm{MM}$ at 2 years $(p$ value $\leq 0.25$ ).

From among these, the stepwise model selection and random forest algorithm identified serum M-protein concentration, involved to uninvolved sFLC ratio, and BMPC percentage as the most relevant factors predicting progression to MM, and based on Youden's Index in the ROC analyses, the optimal cut-offs for the risk factors were $1.9 \mathrm{~g} / \mathrm{dL}$ for serum M-protein (specificity 60\% and sensitivity $71 \%$ ), 19.3 for the involved to uninvolved sFLC ratio (specificity $79 \%$ and sensitivity $55 \%$ ), and $16.4 \%$ for the BMPC infiltration (specificity $55 \%$ and sensitivity $75 \%$ ) as shown in Table 3. For convenience and simplicity, we 


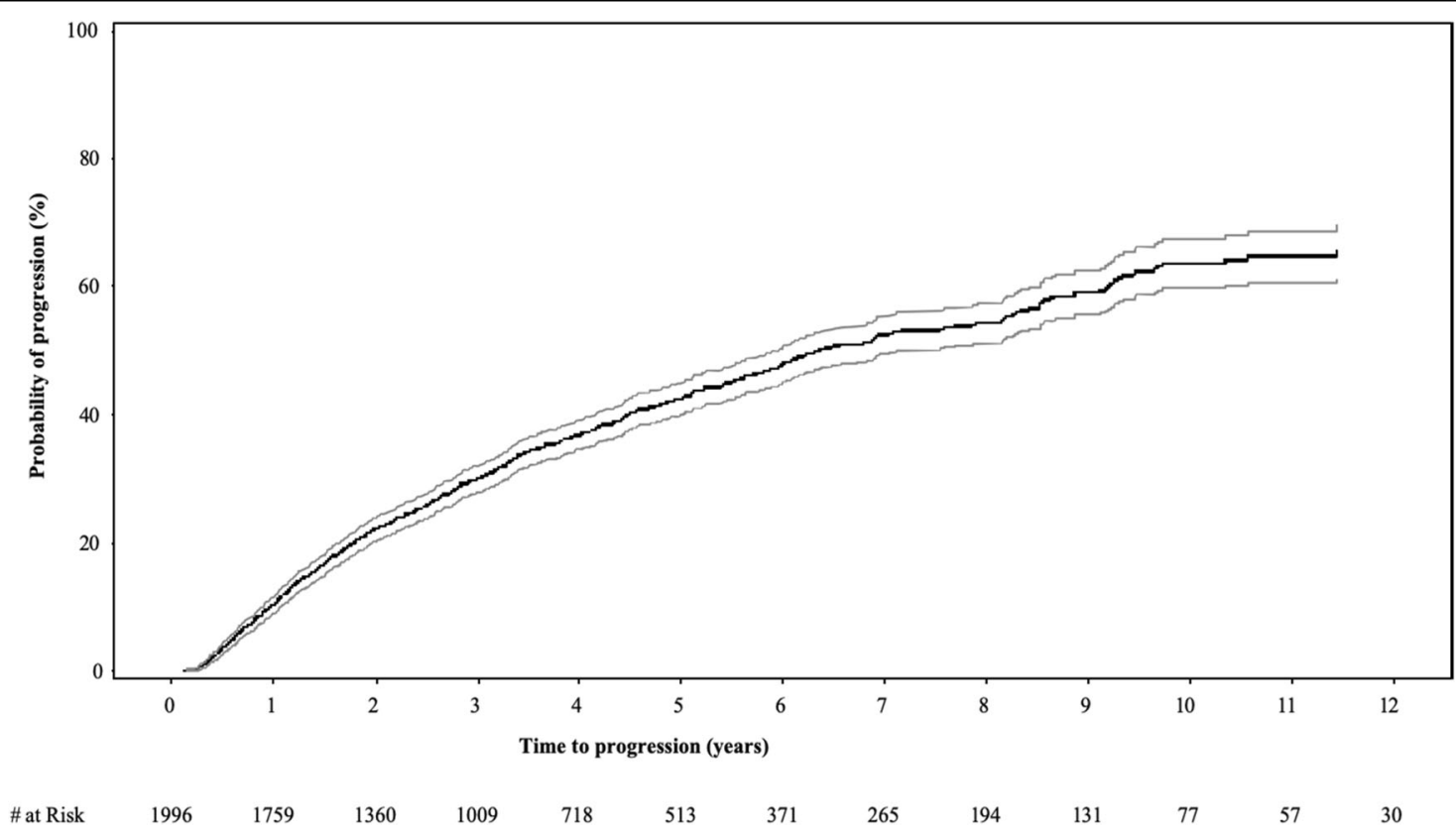

Fig. 1 Probability of progression over time in the full study cohort $(\boldsymbol{n}=\mathbf{1 9 9 6})$. The median time to progression for the entire cohort was 6.4 years (95\% Cl 6.0-7.2); the 2-, 5-, and 10-year risk of progression were 22, 42, and 64\%, respectively.

Table 2 Identification of factors for risk stratification model.

\begin{tabular}{|c|c|c|c|}
\hline & $p$ value & Candidate factors ( $p$ value $<0.25$ ) & Hazard ratio $(95 \% \mathrm{Cl})$ \\
\hline Age (per 10 years) & 0.034 & Yes & $1.09(1.01,1.19)$ \\
\hline Female sex & 0.4552 & No & $0.93(0.77,1.13)$ \\
\hline Hemoglobin (g/dL) & $<0.0001$ & Yes & $0.87(0.82,0.93)$ \\
\hline Creatinine $(\mathrm{mg} / \mathrm{dL})$ & 0.1192 & Yes & $1.2(0.96,1.5)$ \\
\hline Calcium (mg/dL) & 0.6844 & No & $0.98(0.9,1.07)$ \\
\hline Albumin (g/dL) & 0.0786 & Yes & $0.84(0.68,1.02)$ \\
\hline Serum M-protein ( $\mathrm{g} / \mathrm{dL})$ & $<0.0001$ & Yes & $1.09(1.06,1.12)$ \\
\hline Beta-2 microglobulin (mg/dL) & $<0.0001$ & Yes & $1.21(1.14,1.29)$ \\
\hline Absolute difference Kappa-Lambda (mg/dL), per 100 & $<0.0001$ & Yes & $1.03(1.01,1.04)$ \\
\hline Involved to uninvolved sFLC ratio, per 100 & $<0.0001$ & Yes & $1.1(1.06,1.13)$ \\
\hline Heavy-chain type (IgG versus IgM) & 0.5003 & No & $0.92(0.73,1.17)$ \\
\hline Heavy-chain type (IgG versus $\lg A)$ & 0.3336 & No & $0.92(0.78,1.09)$ \\
\hline Light-chain type (Kappa versus Lambda) & 0.0611 & Yes & $0.83(0.68,1.01)$ \\
\hline Immunofixation & 0.9641 & No & $\mathrm{n} / \mathrm{a}$ \\
\hline Immunoparesis $^{\mathrm{a}}$ & $<0.0001$ & Yes & $1.53(1.24,1.89)$ \\
\hline Urine M-spike (mg/24 h), per 1000 & 0.163 & Yes & $1.05(0.98,1.14)$ \\
\hline $\mathrm{BMPC}^{\mathrm{b}} \%$, per 10 & $<0.0001$ & Yes & $1.44(1.36,1.52)$ \\
\hline
\end{tabular}

n/a not applicable.

${ }^{\mathrm{a}}$ Immunoparesis was defined as reduction in one or more of the uninvolved immunoglobulin level below normal range.

${ }^{\mathrm{b}}$ Bone marrow plasma cell percentage 
Table 3 Receiver operating characteristic (ROC) curves with area under the curve (AUC) analysis to identify optimal cut-offs for continuous risk factors (identified from Table 2) with respect to progression to MM within 2 years.

\begin{tabular}{llll}
\hline & Cut point & Specificity (\%) & Sensitivity (\%) \\
\hline Age (per 10 years) & 57 & 28 & 80.6 \\
Hemoglobin (g/dL) & 13.3 & 39.9 & 73.3 \\
Creatinine (mg/dL) & 0.81 & 42.9 & 66 \\
Albumin (g/dL) & 4.09 & 46.4 & 64 \\
Serum M-protein (g/dL) & 1.91 & 60 & 70.6 \\
Beta-2 microglobulin & 2.57 & 65.7 & 52.8 \\
(mg/dL) & & & \\
Absolute difference & 18.5 & 66 & 54.6 \\
$\begin{array}{l}\text { Kappa-Lambda (mg/dL), } \\
\text { per 100 }\end{array}$ & & & \\
Involved to uninvolved & 19.3 & 78.7 & 54.7 \\
SFLC ratio, per 100 & & & 29.8 \\
$\begin{array}{l}\text { Urine M-spike (mg/24 h), } \\
\text { per 1000 }\end{array}$ & & 83.1 & 71.8 \\
$\begin{array}{l}\text { BMPC, higher of biopsy } \\
\text { and aspirate \% }\end{array}$ & 16.4 & 58.1 & \\
\hline
\end{tabular}

decided to use $2 \mathrm{~g} / \mathrm{dL}, 20 \%$, and $20 \%$ as cut-off levels for these factors. Using these cut points, multivariable analysis identified the presence of serum M-protein $>2 \mathrm{~g} / \mathrm{dL}$ as an independent prognostic factor predicting high risk of progression to MM (HR: 2.07, 95\% CI: 1.62-2.65), together with involved to uninvolved sFLC ratio $>20$ (HR: 2.66, 95\% CI: 2.09-3.38) and BMPC infiltration $>20 \%$ (HR: 2.39, 95\% CI: $1.87-3.05$ ).

We then proceeded to create a risk stratification model including 1363 patients in whom all three factors were available: five hundred and twenty-two patients (38\%) did not present any of the factors (reference group) with a risk of progression at 2 years of 6\% (the low-risk group); 1 out of the 3 factors was identified in 445 patients (33\%) with a $18 \%$ risk of progression to MM at 2 years (HR: $2.99,95 \%$ CI: 1.97-4.54) (the intermediate-risk group); and the high-risk group defined by the presence of 2 or 3 risk factors included 396 patients (29\%) with a $44 \%$ progression risk at 2 years (HR: 9.02, 95\% CI: 6.15-13.2) (Fig. 2a). Of the 396 patients included in the high-risk group, 92 presented with the 3 risk factors and had a slightly higher risk of progression to MM (Fig. 2b).

We explored the added value of the presence of any cytogenetic abnormalities to the $2 / 20 / 20$ risk model if all factors were available (689 patients). The stepwise model selection identified the presence of $t(4 ; 14), t(14 ; 16),+1 q$, and del13q/monosomy 13 by fluorescence in situ hybridization (FISH) as the most relevant ones. This model defined four groups of SMM patients with different progression risk at 2 years (Fig. 3): low risk with a progression risk at 2 years of $6 \%(n=225 ; 33 \%)$ and defined by the presence of none of the factors; low-intermediate $(n=$ $224 ; 33 \%)$ if one factor was present and the progression risk at 2 years was $23 \%$ (HR: 4.16, 95\% CI: 2.26-7.67); intermediate risk $(n=177 ; 26 \%)$ defined by the presence of 2 factors and risk of progression at 2 years of $46 \%$ (HR: 9.82, 95\% CI: $5.46-17.7)$; and the high risk $(n=63 ; 9 \%)$ with a progression risk at 2 years of $63 \%$ (HR: 15.5 , 95\% CI: 8.23-29.0) if $\geq 3$ of the factors were present.

\section{Scoring tool to predict risk of progression at 2 years}

Based on the univariate and multivariate analysis previously conducted, the three risk factors (serum M-protein, involved to uninvolved sFLC ratio, and BMPC infiltration) together with the cytogenetic abnormalities were included in a logistic regression model for creating a scoring tool to provide a more individualized assessment of risk. This approach allowed us to utilize the variables along the entire range of values instead of using single cut points. Six hundred and eighty-nine patients were included and Tables 4 and 5 present the score for each risk factor and the predicted risk according to individual risk scores. Patients with total risk score between 0 and 4 had a 2-year progression risk of $3.8 \%$ (reference group). The risk was $26 \%$ (HR: 7.56, 95\% CI: 3.77-15.2) for patients with a total score between 5 and 8 , and for those with a score between 9 and 12, the risk of progression at 2 years was 51\% (HR: 17.3, 95\% CI: 8.63-34.8). When the score was $>12$, the 2 -year progression risk was $73 \%$ (HR: 31.9 , 95\% CI: 15.4-66.3) (Fig. 4).

\section{Discussion}

The treatment paradigms for myeloma has evolved rapidly in the past decade with introduction of new therapies, leading to improved survival ${ }^{18,19}$. The mostly incurable nature of $\mathrm{MM}$ and a better understanding of the disease biology coupled with the prolonged precursor phase has inevitably directed our attention to developing strategies for prevention or early intervention with the goal of delaying onset of active disease or potentially eradicating the clone $\mathrm{e}^{20,21}$. The redefinition of active myeloma was a first step in early intervention, with treatment initiation not solely based on CRAB criteria. The current study fills an important gap in terms of a risk stratification approach for the newly defined SMM that will allow us to design future clinical trials. We present here a simple risk stratification model that can be applied across the globe using easily available data to identify a subgroup of SMM patients with $50 \%$ progression risk at 2 years, defined by the presence of two or three factors 


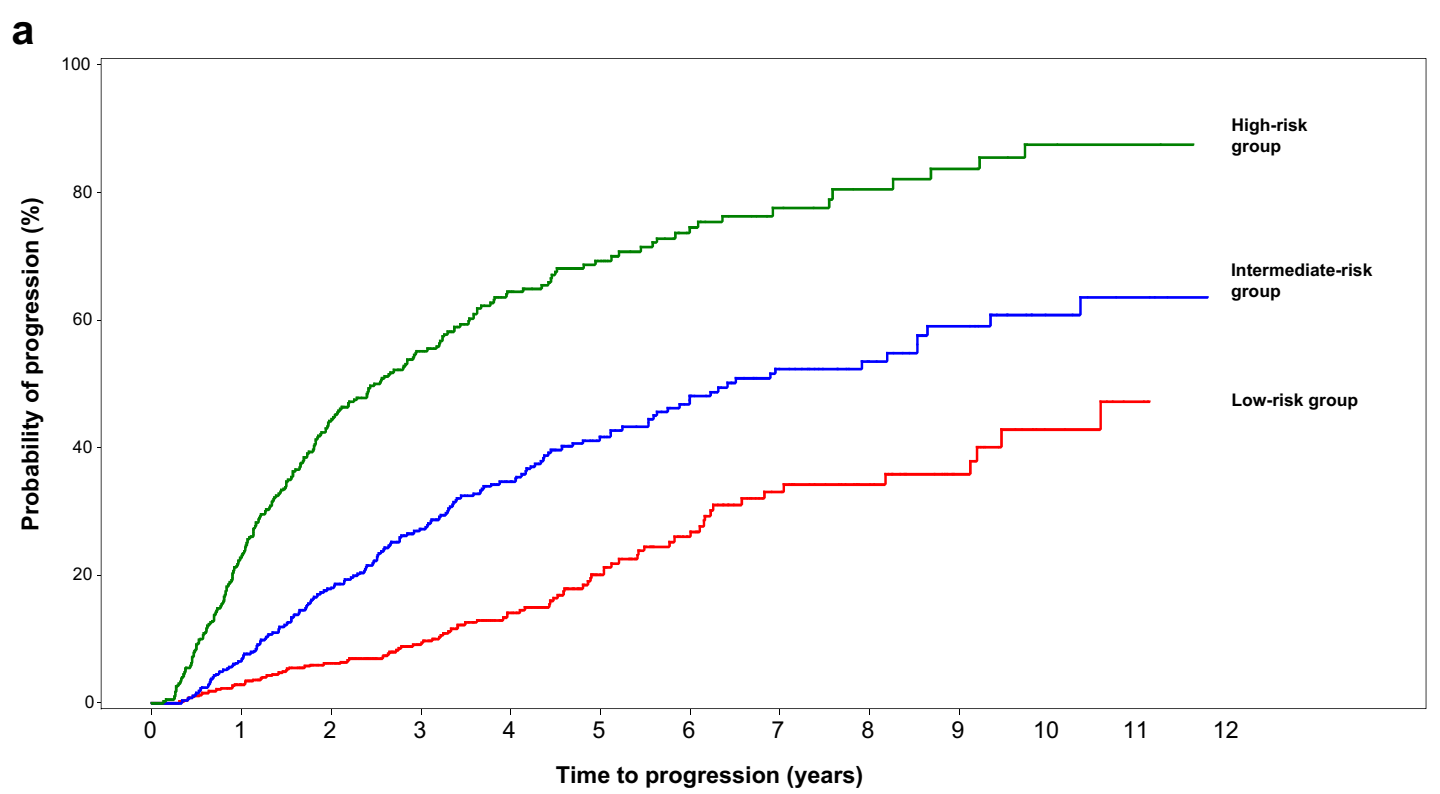

\begin{tabular}{|l|c|c|c|c|}
\hline Risk Stratification groups & Number of risk factors & Hazard Ratio (95\% Cl) & Risk of progression (2 years) & \# of patients \\
\hline Low-Risk & 0 & Reference & $6.2 \%$ & $522(38.3 \%)$ \\
\hline Intermediate & 1 & $2.99(1.97-4.54)$ & $17.9 \%$ & $445(32.7 \%)$ \\
\hline High & $2-3$ & $9.02(6.15-13.2)$ & $44.2 \%$ & $396(29.1 \%)$ \\
\hline
\end{tabular}

b

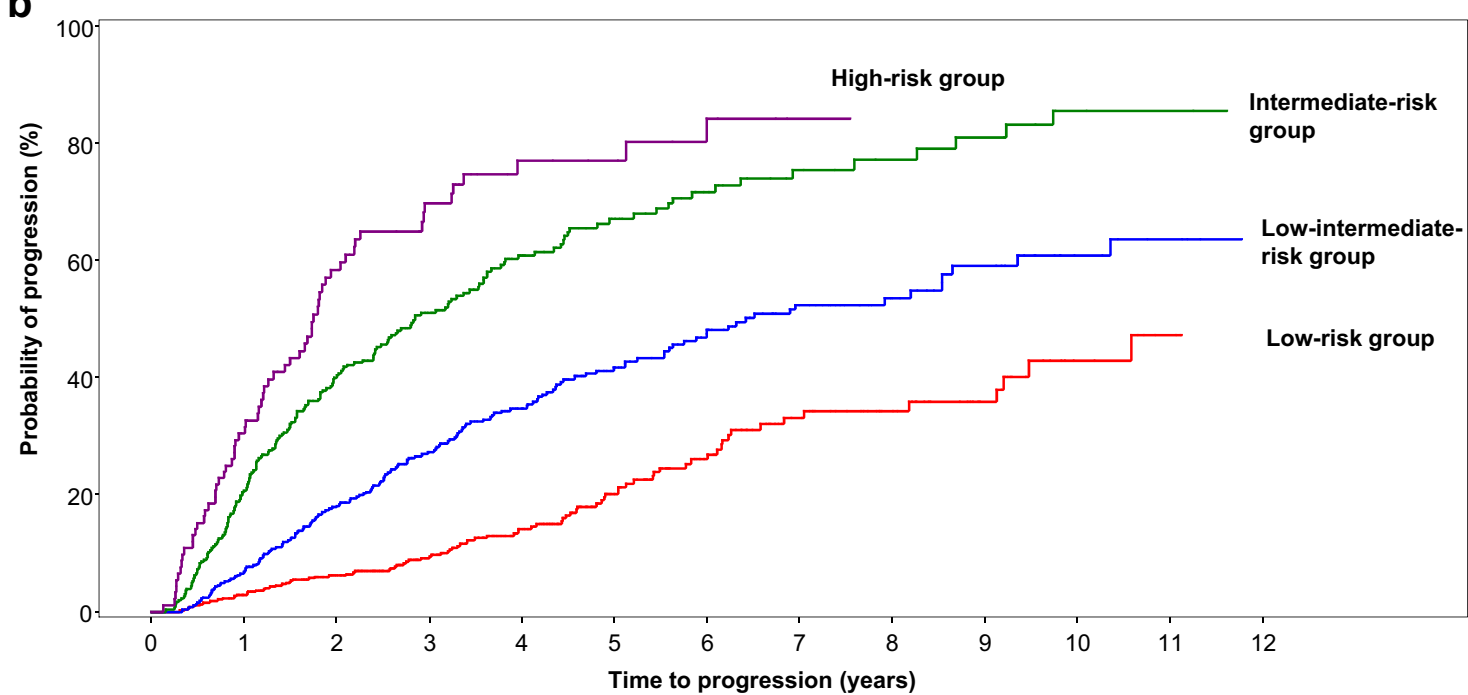

\begin{tabular}{|l|c|c|c|c|}
\hline Risk Stratification groups & Number of risk factors & Hazard Ratio (95\% Cl) & Risk of progression (2 years) & \# of patients \\
\hline Low-Risk & 0 & Reference & $6.2 \%$ & $522(38.3 \%)$ \\
\hline Intermediate & 1 & $2.99(1.97-4.54)$ & $17.9 \%$ & $445(32.7 \%)$ \\
\hline High & $2-3$ & $9.02(6.15-13.2)$ & $44.2 \%$ & $396(29.1 \%)$ \\
\hline
\end{tabular}

Fig. 2 Risk of progression at 2 years based on presence or absence of risk factors in patients with smoldering multiple myeloma. a Probability of progression at 2 years in the three different subgroups of patients according to the model 2/20/20. Patients with no risk factors (low-risk group) had a risk of progression at 2 years of $6 \%$, those with one factor (intermediate-risk group) had a risk of progression of $18 \%$ at 2 years, and those with $\geq 2$ factors (high-risk group) had a 44\% progression risk at 2 years. b Probability of progression at 2 years according to the model 2/20/20 with separation of high-risk group based on presence of 2 or 3 risk factors. Of the 396 patients included in the high-risk group, 92 presented with the three risk factors and had a slightly higher risk of progression to MM. 


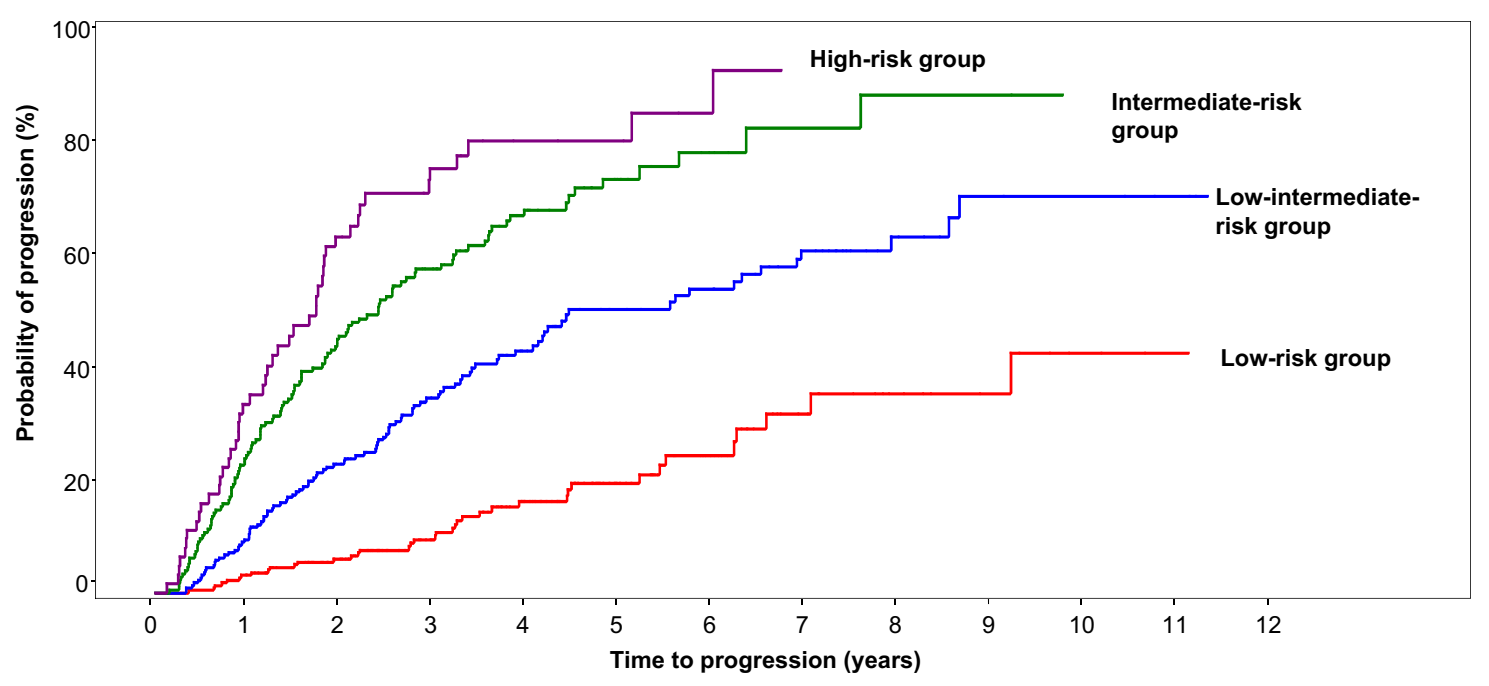

\begin{tabular}{|l|c|c|c|c|}
\hline Risk Stratification groups & Number of risk factors & Hazard Ratio (95\% Cl) & Risk of progression (2 years) & \# of patients \\
\hline Low & 0 & Reference & $6.0 \%$ & $225(32.7 \%)$ \\
\hline Low-intermediate & 1 & $4.16(2.26-7.67)$ & $22.8 \%$ & $224(32.5 \%)$ \\
\hline Intermediate & 2 & $9.82(5.46-17.7)$ & $45.5 \%$ & $177(25.7 \%)$ \\
\hline High & $3-4$ & $15.5(8.23-29.0)$ & $63.1 \%$ & $63(9.1 \%)$ \\
\hline
\end{tabular}

Fig. 3 Probability of progression at 2 years in the four different subgroups of patients according to the model 2/20/20 plus cytogenetic abnormalities $(\mathbf{t}(\mathbf{4} ; \mathbf{1 4}), \mathbf{t}(\mathbf{1 4} ; \mathbf{1 6}),+\mathbf{1 q}$, and/or del13q/monosomy 13$)$. This model defined four groups of SMM patients: low risk with none of the factors had a progression risk at 2 years of $6 \%$, low-intermediate with one factor present had a progression risk at 2 years of $23 \%$, intermediate risk with the presence of 2 factors had a risk of progression at 2 years of $37 \%$, and the high risk with $\geq 3$ of the factors had a progression risk at 2 years of $63 \%$.

among M-protein $>2 \mathrm{~g} / \mathrm{dL}$, BMPCs infiltration $>20 \%$, or the ratio of involved to uninvolved sFLC $>20$. In this study, we used 2-year progression as the end point in order to maintain the approach used in the previous models but now excluding the ultrahigh risk patients with $\geq 80 \%$ risk of progression at 2 years who are essentially active $M$ by current definition. This group represents a relevant population for early intervention and inclusion in trials in order to either prevent the MM development or potentially cure the disease.

The updated IMWG definition segregated from the classical SMM definition those patients with an imminent risk of progression to MM; now considered as MM. This subset of patients could potentially overestimate the progression risk in the classical SMM series and should therefore be excluded from the SMM risk stratification models. The Mayo Clinic group recently examined their cohort of SMM patients by removing those with MM according to the 2014 IMWG criteria, and they identified SMM at $50 \%$ risk of progression at 2 years based on Mprotein $(>2 \mathrm{~g} / \mathrm{dL}), \mathrm{BMPC}$ infiltration $(>20 \%)$, and the ratio of involved to uninvolved sFLC $(>20)^{22}$. Importantly, this international database of nearly 2000 SMM patients confirmed the Mayo Clinic 2/20/20 model, providing robust validation for this new categorization of SMM patients. In addition, the model is based on three features easily available at most centers. Moreover, a subanalysis conducted in a subset of patients with FISH information showed that the presence of any one of the cytogenetic abnormalities such as $\mathrm{t}(4 ; 14), \mathrm{t}(14 ; 16),+1 \mathrm{q}$, or del13q/ monosomy 13 was an additional predictor of progression in this study. This is in line with a previous observation from the Mayo Clinic study that identified the presence of $\mathrm{t}(4 ; 14)$, del17p), and/or hyperdiploidy as predictors of progression ${ }^{10}$. Altogether these data reinforce the concept that the genomic information such as mutations and translocation affecting MYC may contribute to refine the model, but this will require prospective validation in other studies $^{23}$.

Different investigators have defined alternative models to evaluate the risk of progression from SMM to MM. Most have been based on measurements of tumor bulk: size of M-spike, sFLCs, type of immunoglobulin IgA versus IgG, proportion of clonal plasma cells $>95 \%$ of aberrant cells, circulating plasma cells, or immune paresis. These models are useful and have driven the clinical research so far, being validated in clinical trials. However, new models have emerged based on relatively small series 
Table 4 Logistic regression equation to develop the risk score predicting progression risk at 2 years.

\begin{tabular}{lllll}
\hline Risk factor & Coefficient & Odds ratio $(95 \% \mathrm{Cl})$ & $\boldsymbol{p}$ value & Score \\
\hline FLC IU & & & & \\
0-10 (reference) & - & - & - & 0 \\
$>10-25$ & 0.69 & $1.99(1.15,3.45)$ & 0.014 & 2 \\
$>25-40$ & 0.96 & $2.61(1.36,4.99)$ & 0.004 & 3 \\
$>40$ & 1.56 & $4.73(2.88,7.77)$ & $<0.0001$ & 5 \\
M-protein & & & & \\
0-1.5 (reference) & - & - & - & 0 \\
$>1.5-3$ & 0.95 & $2.59(1.56,4.31)$ & 0.0002 & 3 \\
$>3$ & 1.3 & $3.65(2.02,6.61)$ & $<0.0001$ & 4 \\
BMPC & & & & \\
0-15 (reference) & - & - & - & 0 \\
$>15-20$ & 0.57 & $1.77(1.03,3.06)$ & 0.04 & 2 \\
$>20-30$ & 1.01 & $2.74(1.6,4.68)$ & 0.0002 & 3 \\
$>30-40$ & 1.57 & $4.82(2.5,9.28)$ & $<0.0001$ & 5 \\
$>40$ & 2 & $7.42(3.23,17.02)$ & $<0.0001$ & 6 \\
FISH abnormality & 0.83 & $2.28(1.53,3.42)$ & $<0.0001$ & 2 \\
\hline
\end{tabular}

FLC IU involved to uninvolved serum-free light chain ratio. of patients utilizing new imaging assessments like PET$\mathrm{CT}$, genetic signatures, Bence Jones proteinuria, or dynamic models such as the evolution of the Mcomponent and the decrease of hemoglobin, among others. Although many of them identify SMM patients with $\geq 50 \%$ risk of progression to MM within the first 2 years since diagnosis, in clinical practice, physicians are frequently confused about what model to use to define the risk of progression in SMM; moreover, many ongoing clinical trials in SMM use different inclusion criteria, which may be a confounding factor upon analyzing the efficacy of new drugs/combinations in this setting. Taken together, this model derived from an international SMM population with commonly available and reproducible biomarkers could be employed as a standard in registration trials as well as routine clinical practice.

The phase 3 trial conducted by the ECOG group evaluating single-agent lenalidomide versus observation in SMM patients showed a significant benefit in progressionfree survival for the high-risk subset defined as in the current study using the $2 / 20 / 20$ model $^{7}$. Moreover, the same group decided to amend their new phase 3 trial comparing Ld versus Ld plus daratumumab in high-risk SMM in order to introduce the $2 / 20 / 20$ model as inclusion criteria. The ASCENT trial conducted by the

Table 5 Predictive values of risk score tool.

\begin{tabular}{|c|c|c|c|c|c|}
\hline \multirow[t]{2}{*}{ Total risk score } & \multicolumn{2}{|c|}{ Predicted risk at 2 years based on } & \multirow[t]{2}{*}{ Actual (\% with 2-year progression) } & \multicolumn{2}{|c|}{ Predictive value } \\
\hline & Risk score & Full regression model & & Positive & Negative \\
\hline 0 & 3.2 & 3.3 & $1(1.3 \%)$ & 25.8 & $\mathrm{n} / \mathrm{a}$ \\
\hline 2 & 6.2 & 6.1 & $3(5.4 \%)$ & 29.1 & 98.8 \\
\hline 3 & 8.5 & 8.3 & $2(2.6 \%)$ & 31.5 & 97.1 \\
\hline 4 & 11.6 & 11.1 & $3(10.3 \%)$ & 36.1 & 97.2 \\
\hline 5 & 15.7 & 14.8 & 19 (19.2\%) & 37.7 & 96.3 \\
\hline 6 & 20.8 & 19.4 & $11(23.4 \%)$ & 43 & 91.8 \\
\hline 7 & 27 & 25 & $16(27.6 \%)$ & 46 & 89.9 \\
\hline 8 & 34.3 & 31.5 & $21(35 \%)$ & 50.4 & 87.6 \\
\hline 9 & 42.5 & 39 & $17(48.6 \%)$ & 55.4 & 85 \\
\hline 10 & 51 & 46.9 & $18(41.9 \%)$ & 57 & 82.8 \\
\hline 11 & 59.5 & 55 & $17(50 \%)$ & 63.2 & 81 \\
\hline 12 & 67.5 & 62.9 & $13(61.9 \%)$ & 69.4 & 79.3 \\
\hline 13 & 74.6 & 70.1 & $8(50 \%)$ & 72.5 & 77.9 \\
\hline 14 & 80.5 & 76.5 & $11(78.6 \%)$ & 82.9 & 77.2 \\
\hline 15 & 85.4 & 81.8 & $10(83.3 \%)$ & 85.7 & 76 \\
\hline $16+$ & 89.2 & 86.2 & 8 (88.9\%) & 88.9 & 75 \\
\hline
\end{tabular}




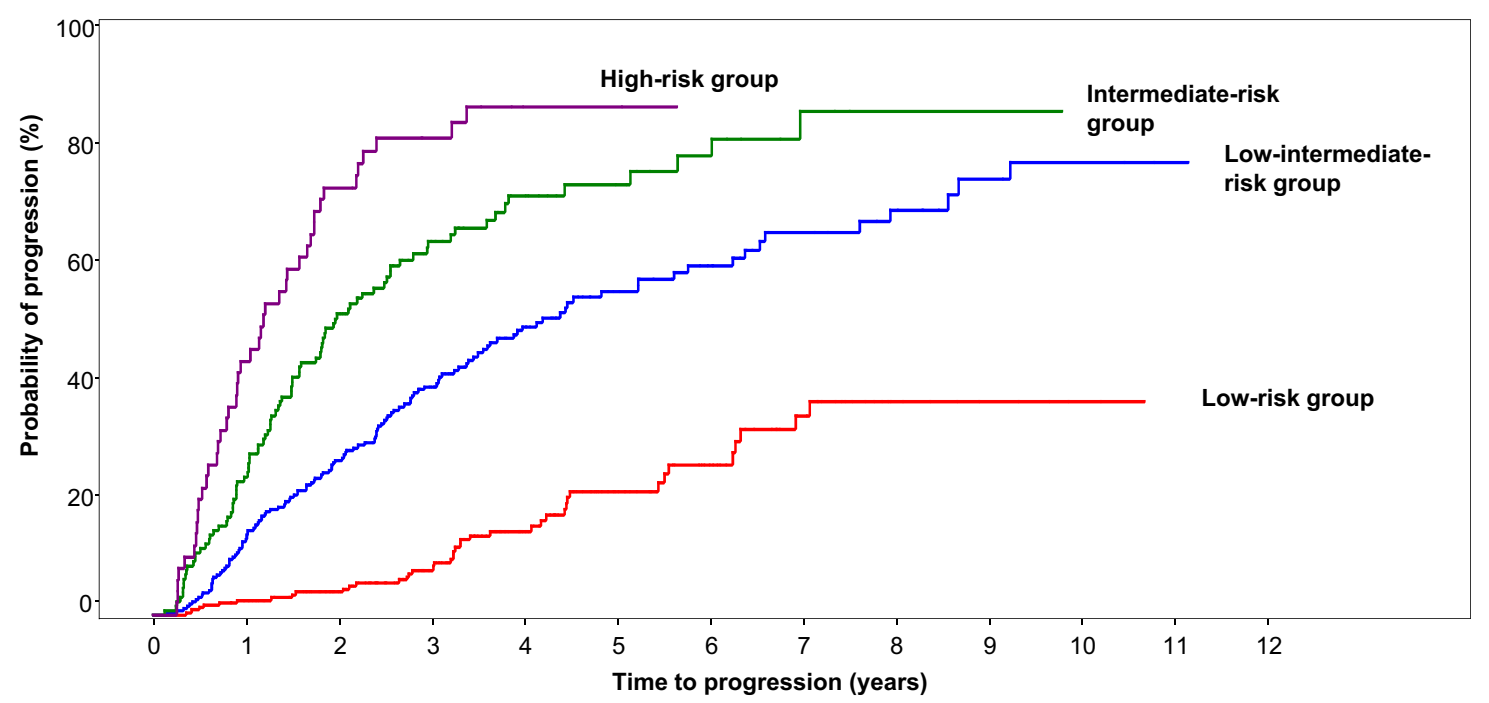

\begin{tabular}{|l|c|c|c|c|}
\hline Risk Stratification groups & Total risk score & Hazard Ratio (95\% Cl) & Risk of progression (2 years) & \# of patients \\
\hline Low & $0-4$ & Reference & $3.8 \%$ & $241(35.0 \%)$ \\
\hline Low-intermediate & $5-8$ & $7.56(3.77-15.2)$ & $26.2 \%$ & $264(38.3 \%)$ \\
\hline Intermediate & $9-12$ & $17.3(8.63-34.8)$ & $51.1 \%$ & $133(19.3 \%)$ \\
\hline High & $>12$ & $31.9(15.4-66.3)$ & $72.5 \%$ & $51(7.4 \%)$ \\
\hline
\end{tabular}

Fig. 4 Risk of progression according to the risk score. Risk score was developed using the entire range of the values for BMPC, serum FLC, and serum M-spike as well as cytogenetic abnormality. Patients with total risk score between 0 and 4 had a 2-year progression risk of 3.8\%, patients with a total score between 5 and 8 had a risk of $26 \%$, those with a score between 9 and 12 had a risk of progression of $51 \%$, and those with a score $>12$ had a risk of progression of $73 \%$.

International Myeloma Foundation with a curative strategy is using the same model.

While we used 2-year progression as the end point to define a high-risk SMM population, we have also created a more precise and individualized scoring tool to classify individuals by risk of progression using the entire spectrum of values for each patient (in place of dichotomous division) including M-spike, BMPC infiltration, and sFLC ratio. Accordingly, this scoring tool is able to precisely identify SMM patients with extremely low risk of progression at 2 years (close to MGUS), as well as SMM with a risk of progression at 2 years even $>50 \%$. Thus, using this risk scoring, SMM patients with total risk score of 1 have $90 \%$ of probability of not developing MM in 2 years (negative predictive value $(\mathrm{NPV})=90 \%$ ), while for those patients with total risk score of 9 , the probability of developing MM in 2 years will be of $93 \%$ (positive predictive value $=93 \%$ ). However, the identification of SMM patients who will not progress with near certainty $(100 \%$ NPV) is difficult, and in the subgroup of patients with total risk score of 0 , the risk does exist.

There are some limitations in this study because of its retrospective nature as well as the missing data observed for some variables that may have led to their exclusion in the multistep process. This is the case for the presence of immune paresis, percentage of plasma cells with aberrant phenotype or circulating plasma cells, or the evolution of the M-component and the decrease in hemoglobin. In addition, differences in the specific methodology used for FISH may vary from institution to institution. One additional limitation is its complete reliance on clinical features. It has been recently shown that the mutational landscape, particularly mutations in the RAS family as well as c-Myc alterations, may independently predict progression risk $^{24}$. Moreover, the transition process from SMM to MM could also involve growth of preexisting clones due to a more permissive bone marrow microenvironment ${ }^{25,26}$. The current study also does not factor in other demographic factors such as race as the numbers were insufficient to explore this.

In summary, our study identifies a subgroup of SMM patients with $50 \%$ progression risk at 2 years from diagnosis based on the presence of two or three factors among M-protein $(>2 \mathrm{~g} / \mathrm{dL})$, BMPC infiltration $(>20 \%)$, or the ratio of involved versus uninvolved sFLC $(>20)$. This model is easily reproducible and available worldwide, could be used to identify high-risk SMM patients in the context of clinical research, and will contribute in the near future to be able to offer early treatment to a more homogeneous subgroup of SMM patients. Availability of 
FISH data can improve the model and should be considered in all patients diagnosed with SMM. In addition, development of a scoring system allows for more individualized risk assessment. The trials going on and incorporating these features for the selection of the high-risk SMM patients will contribute to validate the model and ancillary biological studies can help to optimize it in the future. Future studies should focus on incorporating other tumor cell characteristics such as the presence of mutations as well as alterations in the tumor microenvironment, especially changes in the immune parameters.

\section{Acknowledgements}

We would like to acknowledge Amirah Limayo and Lisa Paik at the International Myeloma Foundation for their contribution and Anja Haltner, Steven Doucette, and Chris at the Cornerstone group for their role in the statistical analysis. The data collection study was conducted by the International Myeloma Foundation as an International Myeloma Working Group project supported in part by a grant funded by Janssen.

\section{Author details}

${ }^{1}$ Servicio de Hematologia, Hospital Universitario de Salamanca, Centro de Investigación del Cáncer, Instituto de Biología Molecular y Cellular del Cáncer (Universitario de Salamanca-Consejo Superior de Investigaciones Científicas), Salamanca, Spain. ²Division of Hematology, Mayo Clinic, Rochester, MN, USA ${ }^{3}$ Hematology and Medical Oncology, Department of Clinical Therapeutics, National and Kapodistrian University of Athens, School of Medicine, Athens, Greece. ${ }^{4}$ Department of HematoOncology, University Hospital Ostrava and Faculty of Medicine, Ostrava University, Ostrava, Czech Republic. ${ }^{5}$ Department of Hematology, Amyloidosis and Myeloma Unit, Hospital Clínic, IDIBAPS, University of Barcelona, Barcelona, Spain. ${ }^{6}$ Perlmutter Cancer Center, NY Langone Health, New York, NY, USA. ${ }^{7}$ Department of Molecular Medicine, Amyloidosis Research and Treatment Center, Foundation 'Istituto di Ricovero e Cura a Carattere Scientifico (IRCCS) Policlinico San Matteo', University of Pavia, Pavia, Italy. ${ }^{8}$ Department of Internal Medicine V, University Medical Hospital and National Center of Tumor Diseases, Heidelberg, Germany. ${ }^{9}$ Department of Clinical Hematology, Centro Hospitalar e Universitário de Coimbra, Coimbra, Portugal. ${ }^{10}$ Department of Medicine, Surgery and Neurosciences, University of Siena, Siena, Italy. ${ }^{11}$ Department of Haematology, London North West Healthcare, London, UK. ${ }^{12}$ Service d'Hématologie, Hôpital Pitié Salpêtrière, Paris, France. ${ }^{13}$ Department of Hematology, Sahlgrenska University Hospital, Goteborg and Skane University Hospital, Lund, Sweden. ${ }^{14}$ Department of Experimental, Diagnostic and Specialty Medicine - DIMES, University of Bologna, Bologna, Italy. ${ }^{15}$ Department of Hematology, Hospital Italiano de Buenos Aires, Buenos Aires, Argentina. ${ }^{16}$ Department of Hematology, Hopital de La Milétrie, CHU, Poitiers, France. ${ }^{17}$ Department of Hemato-Oncology, Hallym University Dongtan Sacred Heart Hospital, Hwasung, South Korea. ${ }^{18}$ Department of Hematology, Hospital de Santa Maria, Lisboa, Portugal. ${ }^{19}$ Department of Medicine, Center for Oncology, Hematology and Palliative Care, Wilhelminenspital, Vienna, Austria. ${ }^{20}$ Department of Hematology and Medical Oncology, Atrium Health/Levine Cancer Institute, Charlotte, NC, USA. ${ }^{21}$ Hematology, Department of Internal Medicine, Seoul St. Mary's Hospital, The Catholic University of Korea, Seoul, South Korea. ${ }^{22}$ Janssen Pharmaceuticals, Horsham, PA, USA. ${ }^{23}$ Cedars-Sinai Outpatient Cancer Center, Los Angeles, CA, USA. ${ }^{24}$ Clinica Universidad de Navarra, CIMA, CIBERONC, IDISNA, Pamplona, Spain

\section{Author contributions}

M.-V.M. and S.K. designed the study, directed the analysis, and wrote the manuscript. J.S.-M., B.G.M.D., and S.V.R. were involved in the study design and analyses. M.A.D., V.G.-C., E.K., R.H., C.F.d.L., G.J.M., G.M., H.G., C.G., A.G., C.K., L.G., M.H., E.Z., D.F., X.L., B.-S.K., G.E., H.L., S.U., and C.-K.M. enrolled patients and contributed to the manuscript writing. M.Q., J.U., and B.M.W. were involved with study design and manuscript review. The first two (M.-V.M., S.K.) and last two authors (B.G.M.D., J.S.-M.) had full access to all the data in the study. All authors accepted responsibility for the decision to submit the findings for publication.

\section{Conflict of interest}

M.-V.M. reports grants from Janssen, Celgene, Amgen, Takeda, Abbvie, GSK, Adaptive, EDO Mundipharma, Pharmamar, Roche, Seattle Genetics, outside the submitted work; S.K. reports research funding for clinical trials to the institution: Celgene, Takeda, Janssen, BMS, KITE, Merck, AbbVie, Medimmune, Novartis, Roche-Genentech, Amgen, Tenebio, Carsgen; and Consulting/ Advisory Board participation: (with no personal payments) Celgene, Takeda, Janssen, AbbVie, Genentech, Amgen, Molecular Partners and (with personal payment) Oncopeptides, Gene Centrix, Cellectar. M.A.D. reports personal fees from Amgen, Celgene, Takeda, Janssen, and BMS, outside the submitted work. V.G.-C. reports grants from Janssen, personal fees from Advisory Board of Prothena, non-financial support from Janssen and Celgene, outside the submitted work; E.K. reports grants and personal fees from Amgen, personal fees and non-financial support from Genesis Pharma, grants, personal fees, and non-financial support from Janssen, personal fees from Takeda and Pfizer, during the conduct of the study; R.H. has nothing to disclose. C.F.d.L. reports grants, personal fees, and non-financial support from Janssen, Celgene, Takeda, and Amgen, outside the submitted work; G.J.M.: Advisory Boards from Celgene, BMS, Sanofi, Karyopharm, Janssen, Roche, and Genentech. G.M. has nothing to disclose. H.G. reports grants, personal fees and other fees from Celgene, Janssen, BMS, and Sanofi, grants and other from Chugai, grants from John Hopkins University and Dietmar Hopp Stiftung, other fees from Amgen, Molecular Partners, MSD, Mundipharma, and Art Tempi, personal fees and other fees from Takeda and Novartis, nonfinancial support and other fees from Adaptive Biotechnology, outside the submitted work. C.G. has nothing to disclose. A.G. reports grants and personal fees from Janssen, personal fees from Celgene, Amgen, and Takeda, outside the submitted work; C.K. has nothing to disclose. L.G. has nothing to disclose. M.H. has nothing to disclose. E.Z.: Advisory Board for Sanofi, BMS, Janssen, and Takeda. D.F. reports personal fees from Amgen, Janssen, Varifarma, Tecnofarma, Takeda, Sanofi, Glaxo, and Bristol Myers, outside the submitted work; X.L. has nothing to disclose. B.-S.K. reports grants from National Research Foundation of Korea (NRF), during the conduct of the study; G.E. has nothing to disclose. H.L. reports grants from Amgen, Takeda, personal fees from Amgen, Takeda, Janssen, BMS, Celgene, Seattle Genetics, and Sanofi, outside the submitted work; S.U. reports grants and personal fees from Amgen, Celgene, Sanofi, Seattle Genetics, Janssen, Takeda, SkylineDX, and Merck, personal fees from Abbvie and MundiPharma, grants from BMS and Pharmacyclics, outside the submitted work; C.-K.M. has nothing to disclose. M.Q.: employment from Janssen Research and Development; stock or other ownership from Janssen Research and Development. J.U.: employment from Janssen; stock or other ownership from Janssen. B.M.W. is an employee of Janssen Research \& Development. S. V.R. has nothing to disclose. B.G.M.D.: Advisory Board for Amgen, Janssen, Celgene/BMS, and Takeda. J.S.-M. reports other fees from Amgen, BMS, Celgene, Janssen, MSD, Novartis, Takeda, Sanofi, Roche, GSK, AbbVie, and Karyopharm, outside the submitted work.

\section{Publisher's note}

Springer Nature remains neutral with regard to jurisdictional claims in published maps and institutional affiliations.

Received: 8 June 2020 Revised: 16 September 2020 Accepted: 22 September 2020

Published online: 16 October 2020

\section{References}

1. Kyle, R. A. \& Greipp, P. R. Smoldering multiple myeloma. N. Engl. J. Med. 302, 1347-1349 (1980)

2. Rajkumar, S. V., Landgren, O. \& Mateos, M. V. Smoldering multiple myeloma. Blood 125, 3069-3075 (2015).

3. Kyle, R. A. et al. Clinical course and prognosis of smoldering (asymptomatic) multiple myeloma. N. Engl. J. Med. 356, 2582-2590 (2007).

4. Dispenzieri, A. et al. Immunoglobulin free light chain ratio is an independent risk factor for progression of smoldering (asymptomatic) multiple myeloma. Blood 111, 785-789 (2008)

5. Perez-Persona, E. et al. New criteria to identify risk of progression in monoclonal gammopathy of uncertain significance and smoldering multiple 
myeloma based on multiparameter flow cytometry analysis of bone marrow plasma cells. Blood 110, 2586-2592 (2007).

6. Mateos, M. V. et al. Lenalidomide plus dexamethasone for high-risk smoldering multiple myeloma. N. Engl. J. Med. 369, 438-447 (2013).

7. Lonial, S. et al. E3A06: Randomized phase III trial of lenalidomide versus observation alone in patients with asymptomatic high-risk smoldering multiple myeloma. J. Clin. Oncol. 37, 8001-8001 (2019).

8. Rajkumar, S. V. et al. International Myeloma Working Group updated criteria for the diagnosis of multiple myeloma. Lancet Oncol. 15, e538-e548 (2014).

9. Zamagni, E. et al. 18F-FDG PET/CT focal, but not osteolytic, lesions predict the progression of smoldering myeloma to active disease. Leukemia $\mathbf{3 0}, 417-422$ (2016).

10. Rajkumar, S. V. et al. Impact of primary molecular cytogenetic abnormalities and risk of progression in smoldering multiple myeloma. Leukemia 27, 1738-1744 (2013).

11. Fernandez de Larrea, C. et al. Evolving M-protein pattern in patients with smoldering multiple myeloma: impact on early progression. Leukemia $\mathbf{3 2}$ 1427-1434 (2018)

12. Ravi, P. et al. Changes in uninvolved immunoglobulins during induction therapy for newly diagnosed multiple myeloma. Blood Cancer J. 7, e569 (2017).

13. Gonzalez-Calle, V. et al. Bence Jones proteinuria in smoldering multiple myeloma as a predictor marker of progression to symptomatic multiple myeloma. Leukemia 30, 2026-2031 (2016).

14. Dhodapkar, M. V. et al. Clinical, genomic, and imaging predictors of myeloma progression from asymptomatic monoclonal gammopathies (SWOG S0120). Blood 123, 78-85 (2014).

15. Breiman, L. Random forests. Mach. Learn. 45, 5-32 (2001).
16. Svetnik, V. et al. Random forest: a classification and regression tool for compound classification and QSAR modeling. J. Chem. Inf. Comp. Sci. 43, 1947-1958 (2003).

17. Massaro, J. M. et al. Managing and analysing data from a large-scale study on Framingham offspring relating brain structure to cognitive function. Stat. Med. 23, 351-367 (2004)

18. Kyle, R. A. \& Rajkumar, S. V. Multiple myeloma. N. Engl. J. Med. 351, 1860-1873 (2004).

19. Kumar, S. K. \& Rajkumar, S. V. The multiple myelomas - current concepts in cytogenetic classification and therapy. Nat. Rev. Clin. Oncol. 15, 409-421 (2018).

20. Witzig, T. E. et al. A phase III randomized trial of thalidomide plus zoledronic acid versus zoledronic acid alone in patients with asymptomatic multiple myeloma. Leukemia 27, 220-225 (2013).

21. Rajkumar, S. V. et al. Thalidomide as initial therapy for early-stage myeloma Leukemia 17, 775-779 (2003)

22. Lakshman, A. et al. Risk stratification of smoldering multiple myeloma incorporating revised IMWG diagnostic criteria. Blood Cancer J. 8, 59 (2018).

23. Misund, K. et al. MYC dysregulation in the progression of multiple myeloma. Leukemia 34, 322-326 (2020)

24. Bustoros, M. et al. Genomic profiling of smoldering multiple myeloma identifies patients at a high risk of disease progression. J. Clin. Oncol. 38, 2380-2389 (2020).

25. Manier, S., Kawano, Y., Bianchi, G., Roccaro, A. M. \& Ghobrial, I. M. Cell autonomous and microenvironmental regulation of tumor progression in precursor states of multiple myeloma. Curr. Opin. Hematol. 23, 426-433 (2016).

26. Dhodapkar, M. V. MGUS to myeloma: a mysterious gammopathy of underexplored significance. Blood 128, 2599-2606 (2016). 